\title{
Recent results in the deuteron break-up with high momentum transfer at COSY
}

\section{S. Dymov* for the ANKE collaboration}

University of Ferrara and INFN, I-44100 Ferrara, Italy,

Laboratory of Nuclear Problems, JINR, RU-141980 Dubna, Russia

E-mail: s.dymov@fz-juelich.de

\begin{abstract}
The deuteron break-up by proton with high momentum transfer $p d \rightarrow\{p p\}_{s} n$, where $\{p p\}_{s}$ is the proton pair in ${ }^{1} S_{o}$ state has been studied with a polarized COSY proton beam and an unpolarized deterium target at the beam energy of $353 \mathrm{MeV}$. The obtained differential cross section and the proton analysing power are compared to a calculation carried out within $\mathrm{ONE}+\Delta+\mathrm{SS}$ model.
\end{abstract}

23rd International Spin Physics Symposium - SPIN2018 -

10-14 September, 2018

Ferrara, Italy

\footnotetext{
* Speaker.
} 


\section{Introduction}

The hadron reactions with the production of a diproton final state, which is a proton pair $\{p p\}_{s}$ with small excitation energy, have been studied extensively at ANKE-COSY [1]. These included the break-up process $p d \rightarrow\{p p\}_{s} n$ at high and low momentum transfer, the $p N \rightarrow\{p p\}_{s} \pi$ one pion production, the $p p \rightarrow\{p p\}_{s} \gamma$ reaction and study of the $\mathrm{ABC}$ effect in the $p p \rightarrow\{p p\}_{s} \pi \pi$ reaction. The low excitation energy ensures the final $p p$-pair to be in the ${ }^{1} S_{0}$ state, thus reducing the number of the partial waves accessible and simplifying the theoretical description of the process. The hard break-up process $p d \rightarrow\{p p\}_{s} n$ in the colinear kinematics has been studied at ANKE in the energy range $0.5-2.0 \mathrm{GeV}$ [2], where the main reaction mechanisms are the one nucleon exchange (ONE), $\Delta$-excitation and the single scattering (SS). Selection of a diproton in the final state suppresses the $\Delta$ mechanism, allowing one to probe the deuteron structure at short distances. The obtained results, that included the differential cross-section and the vector analysing power, were compared to the model predictions produced with several modern $N N$-potentials. Recently, this study was complemented by the data on the cross section and $A_{y}$ at $353 \mathrm{MeV}$, where one expects the dominance of ONE mechanism, what provides an accurate test of the ONE+ $++\mathrm{SS}$ model [3, 4]. These results, analysed together with the ANKE data on the $p n \rightarrow\{p p\}_{s} \pi^{-}$and $p p \rightarrow\{p p\}_{s} \pi^{0}$ reactions, previously extracted at the same energy, may solve the existing ambiguity in the partial wave analysis of the pion production processes [5].

\section{Experimental setup}

The experiment was carried out using the ANKE magnetic spectrometer [1] positioned inside the COSY storage ring [6] of the Forschungszentrum Jülich. Although the facility sketched in Fig. 1 was equipped with other elements, the only detectors used in this experiment were the forward detector (FD) and the silicon tracking telescopes (STT) [7].

The fast final state protons from the reaction $p d \rightarrow\{p p\}_{s} n$ were measured in the FD in the range $0^{\circ}-15^{\circ}$ in laboratory polar angle $\left(\theta_{\mathrm{lab}}\right)$. The forward detector comprises a set of multiwire proportional and drift chambers and a two-plane scintillation hodoscope. In addition to their use for triggering, the scintillators were also needed to measure the energy losses required for particle identification [8].

The two STT were installed in the COSY plane symmetrically inside the vacuum chamber to the left and right of the beam at distances of $3 \mathrm{~cm}$ from the deuterium cluster-jet target, which had a diameter of the jet of about $1 \mathrm{~cm}$. Each telescope consists of three position-sensitive silicon layers of $70 \mu \mathrm{m}, 300 \mu \mathrm{m}$, and $5 \mathrm{~mm}$ thickness and, in this configuration, covered laboratory polar angles $75^{\circ}<\theta_{\text {lab }}<140^{\circ}$. The acceptances of the STT in azimuth of $\pm 30^{\circ}$ were centered at $\phi=0^{\circ}$ and $\phi=180^{\circ}$ on the left and the right sides, respectively.

The ANKE experiment used a vertically polarized beam incident on an unpolarised target. The sign of the polarization was flipped at every beam injection at the beginning of the acceleration cycle. The quasi-free $p n \rightarrow d \pi^{0}$ process, whose cross section and analysing power are well established at this energy, was used for determination of the luminosity and the beam polarisation. The experiment was carried out at the proton beam energy of $353 \mathrm{MeV}$. 


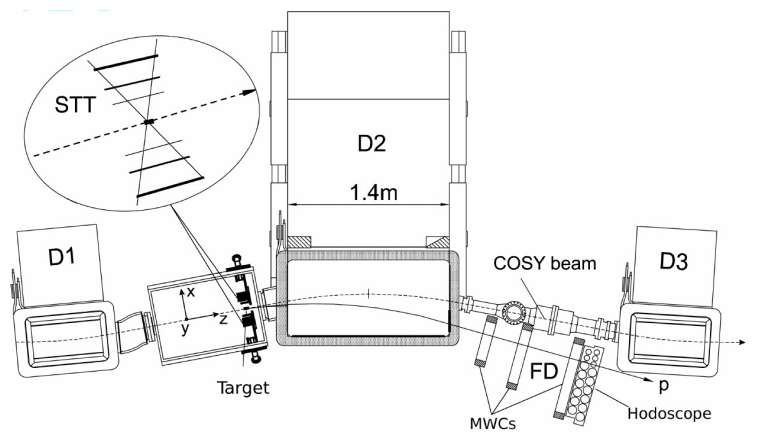

Figure 1: The ANKE spectrometer setup (top view), showing the positions of the deuterium clusterjet target, the silicon tracking telescopes (STT), and the forward detector (FD).

\section{Results}

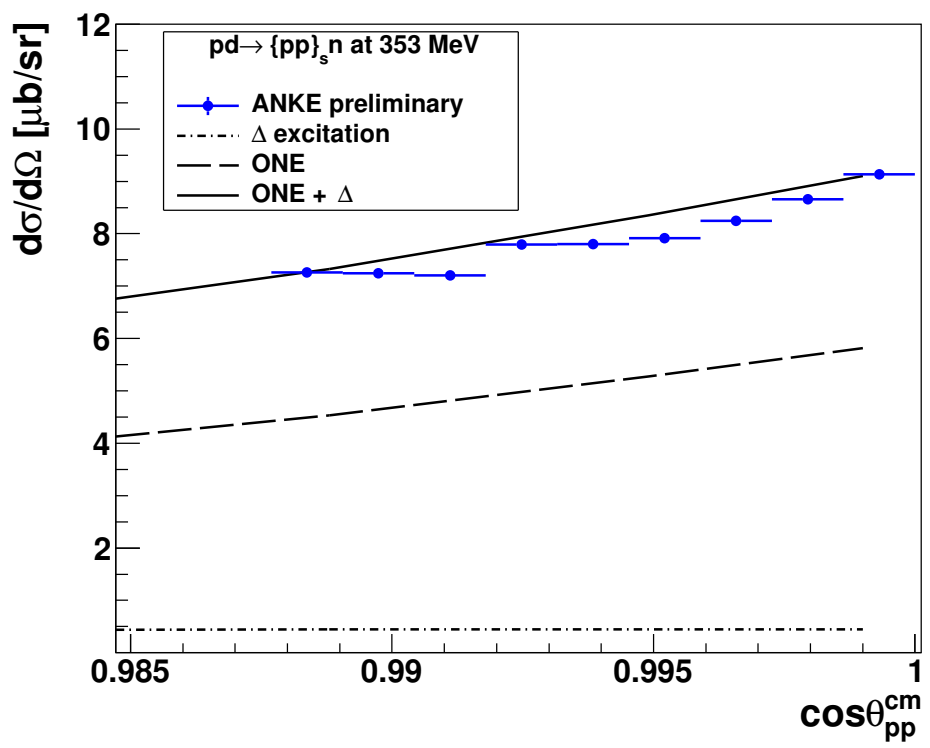

Figure 2: Differential cross section of the $p d \rightarrow\{p p\}_{s} n$ process at $353 \mathrm{MeV}$ as a function of cosine of the $\{p p\}_{s} n$ pair emission angle in the reaction center-of-mass system. The ANKE data (preliminary) are shown with the error bars, the curves present model calculation for the mechanisms ONE and $\Delta$ as noted in the legend.

The results for the differential cross section are shown in Fig. 2, where the preliminary ANKE data are compared to the calculations done within the framework of the ONE+ $\Delta+\mathrm{SS}$ model [9]. The data show good agreement with the model predicting the dominance of the ONE mechanism at the energy of the experiment. In Fig. 3 we show corresponding results for the proton vector analysing power in the process. The measured $A_{y}$ reaches significant level of the absolute value in spite of a very limited angular range covered. Both ONE and $\Delta$ mechanisms alone produce nearly 
zero values of $A_{y}$, and any signal is expected to arise only from their interference. The deviation from data observed can be attributed to the uncertainty in the spin structure of $\Delta$ contribution.

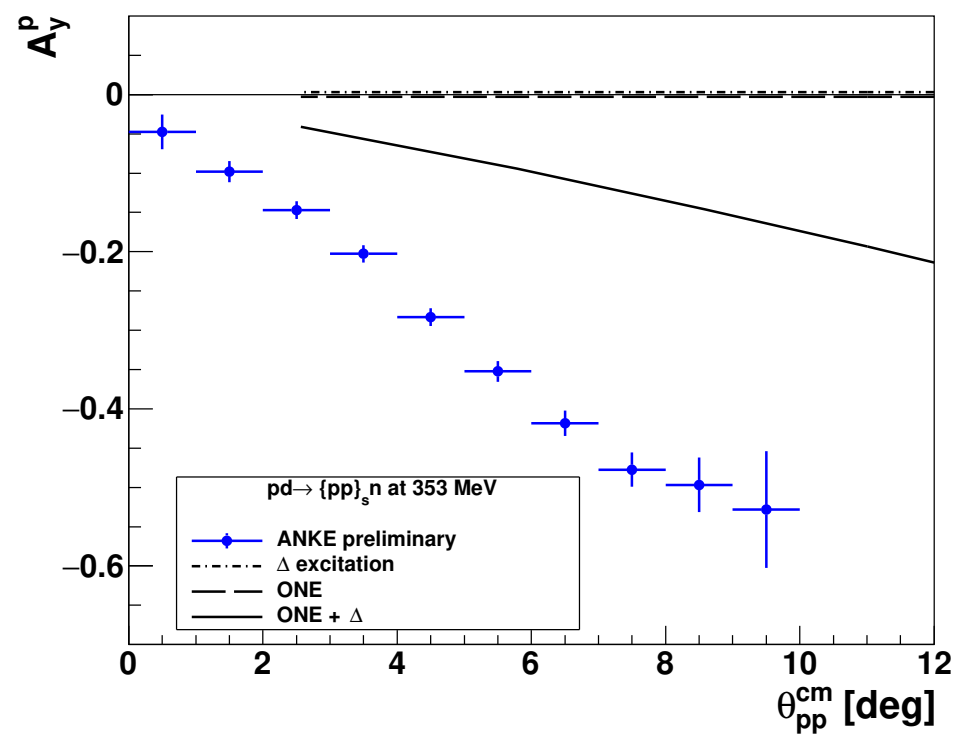

Figure 3: Proton analyzing power of the $p d \rightarrow\{p p\}_{s} n$ process at $353 \mathrm{MeV}$ as a function of the $\{p p\}_{s} n$ pair emission angle in the reaction center-of-mass system. The ANKE data (preliminary) are shown with the error bars, the curves present model calculation for the mechanisms ONE and $\Delta$ as noted in the legend.

\section{References}

[1] S. Barsov et al., ANKE, a new facility for medium energy hadron physics at COSY-Jülich, Nucl. Instrum. Methods Phys. Res. A 462 (2001) 364.

[2] S. Dymov et al., Deuteron breakup $p d \rightarrow\{p p\}_{s} n$ with forward emission of a fast ${ }^{1} S_{0}$ diproton, Phys. Rev. C 81 (2010) 044001.

[3] O. Imambekov and Yu.N. Uzikov, Yad. Fiz. 52 (1990). 1361 [Sov. J. Nucl. Phys. 52 (1990) 862].

[4] Yu.N. Uzikov, J. Phys. G: Nucl. Part. Phys. 28 (2002) B13.

[5] S.Dymov et al., Measurement of spin observables in the quasi-free $n p \rightarrow\{p p\}_{s} \pi-$ reaction at 353 MeV, Phys. Rev. C 88 (2013) 014001.

[6] R. Maier et al., Cooler synchrotron COSY - Performance and perspectives, Nucl. Instrum. Methods Phys. Res. A 390 (1997) 1.

[7] R. Schleichert et al., A self-triggering silicon-tracking telescope for spectator proton detection, IEEE Trans. Nucl. Sci. 50 (2003) 301.

[8] S. Dymov et al., The forward detector of the ANKE spectrometer. Tracking system and its use in data analysis, Part. Nucl. Lett. 2(119) (2004) 40.

[9] Yu. Uzikov, private communications. 